\title{
Posterior Talar Intraosseous Ganglion Treated with Arthroscopic Debridement, Microfracture and Injectable Bone Graft Substitute
}

Roland Walker*, William J Nash, Rajiv S Patel and Sam Singh

Orthopaedic Department, Guy's Hospital, Great Maze Pond, London, UK

\begin{abstract}
We report the case of a 57 year old female patient presenting with longstanding diffuse ankle pain. SPECT-CT was useful in making the diagnosis of an intraosseous ganglion of the posterior talus. The patient was treated with posterior arthroscopic debridement of the cyst via a two portal approach and grafting with an injectable synthetic bone graft substitute (PRO-DENSE $囚)$. The patient experienced rapid relief of pain and made a quick recovery. The surgical technique is discussed as well as a review of the current literature.
\end{abstract}

Keywords: Intraosseous ganglion; Talus; Arthroscopy; Bone graft

\section{Introduction}

Simple, benign cystic lesions of the talus are relatively rare and must be differentiated from degenerative cystic lesions associated with osteochondral injury or established osteoarthritis. The diagnoses include unicameral bone cyst, more commonly seen in children, and intraosseous ganglion seen more commonly in middle-aged adults and first described by Hicks in 1956 [1]. Patients may often attribute the associated ankle pain to a previous minor injury and this may erroneously lead the clinician toward treating a ligamentous injury.

The gold standard surgical management of a talarintraosseous ganglion has been open debridement, curettage and bone grafting with good result [2-4]. More recently, arthroscopic treatment of a variety of ankle conditions has gained popularity and widespread acceptance due to the reduced morbidity and more rapid recovery associated with the technique. The method of hind foot arthroscopy popularized by van Dijk gives good access to the posterior talus and offers an attractive surgical approach to cysts of the posterior talus [5]. Three authors have so far reported their experience treating posterior talarintraosseous ganglions with posterior arthroscopy [6-8]. This is the first case report combining this technique with an injectable synthetic bone graft substitute containing a combination of calcium sulphate and calcium phosphate, designed specifically for the treatment of these types of defects.

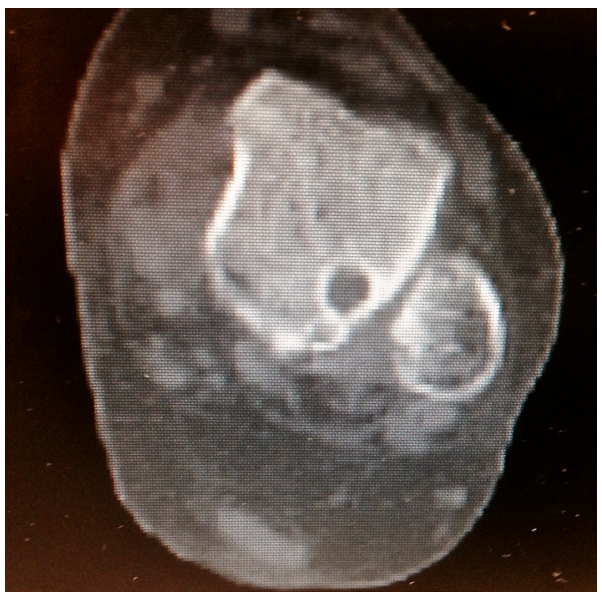

Figure 1: Axial CT showing cyst in posterior talar dome.

\section{Case Report}

A 57 year old female patient presented with longstanding ankle pain, after a mild ankle sprain some years previously. She had failed conservative treatment options with NSAIDs, physiotherapy and bracing and had been recommended a range of surgical options before presenting to the senior author for a second opinion.

Clinical examination revealed some swelling of the ankle, quite generalized tenderness both anteriorly and posteriorly. Anterior draw test was negative and there was clinical evidence of posterior impingement with pain on forced plantar flexion of the ankle. Previous CT and MRI scans revealed a large posterior talar cyst (Figure 1), likely to be an intraosseous ganglion; a small ostrigonum and thickening of the posterior tibiofibular ligament with partial tear and a small avulsion fragment. The ankle joint space was preserved, with no radiological evidence of a chondral or osteochondral lesion. Given she reported pain in 3 different areas of the ankle, to help clarify which pathology was the main pain generator, a SPECT CT scan was requested. This revealed high signal around the talarintraosseous ganglion only (Figure 2) and

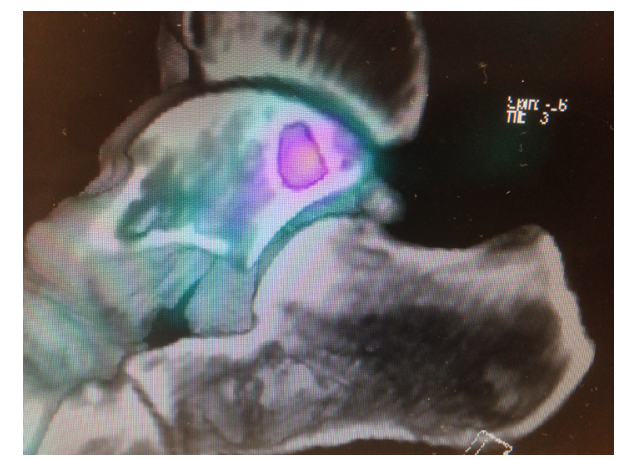

Figure 2: Sagittal SPECT CT showing metabolic activity in cyst.

*Corresponding author: Roland Walker, FRCS (Tr\&Orth), Foot \& Ankle Fellow Orthopaedic Department, Guy's Hospital, Great Maze Pond, London, SE1 9RT, UK, Tel: +44 207188 7188; E-mail: rsrpatel@doctors.org.uk

Received May 28, 2014; Accepted August 07, 2014; Published August 09, 2014

Citation: Walker R, Nash WJ, Patel RS, Singh S (2014) Posterior Talar Intraosseous Ganglion Treated with Arthroscopic Debridement, Microfracture and Injectable Bone Graft Substitute. J Sports Med Doping Stud 4: 143. doi:10.4172/21610673.1000143

Copyright: ( 2014 Walker R, et al. This is an open-access article distributed under the terms of the Creative Commons Attribution License, which permits unrestricted use, distribution, and reproduction in any medium, provided the original author and source are credited. 
Citation: Walker R, Nash WJ, Patel RS, Singh S (2014) Posterior Talar Intraosseous Ganglion Treated with Arthroscopic Debridement, Microfracture and Injectable Bone Graft Substitute. J Sports Med Doping Stud 4: 143. doi:10.4172/2161-0673.1000143

confirmed the clinical suspicion that the cyst was the most likely cause of the patient's ongoing ankle pain. She was advised that arthroscopic surgery to decompress, microfracture and bone graft the cyst would be most likely to address her pain given other simpler non-surgical modalities had been exhausted.

In theatre a tourniquet was placed around the thigh. The patient was positioned in the recovery position with the affected side lowermost and the foot just off the end of the operating table. This position gives excellent access to the posterior ankle and is used by the senior author for posterior ankle arthroscopy as well as Achilles tendon surgery. The limb was exsanguinated and the tourniquet inflated. Two arthroscopy portals were made either side of the Achilles tendon, to access the ankle and subtalar joint, following the principles described van Dijk [5]. The retrocalcaneal fat pad was carefully incised to create a working space and the FHL tendon and the small ostrigonum were then identified as key landmarks. Cross sectional imaging had shown that the posterior talarintraosseous ganglion lay just deep to the small ostrigonum. The small ostrigonum was therefore resected, exposing the posterior ankle joint and the subtalar joint. The cyst revealed itself as a pinhole opening in the posterior talus (Figure 3 ) and the opening was dilated revealing a fibrous tissue membrane lining the cyst (Figure 4). This was easily and completely excised with arthroscopic curettes. Once the floor of the cyst had been exposed a $1.2 \mathrm{~mm}$ kirschner wire was used to create a series of holes into the cancellous bone beneath (Figure 5) in order to release osteoprogenitor cells into the cyst cavity. The cavity was then filled with

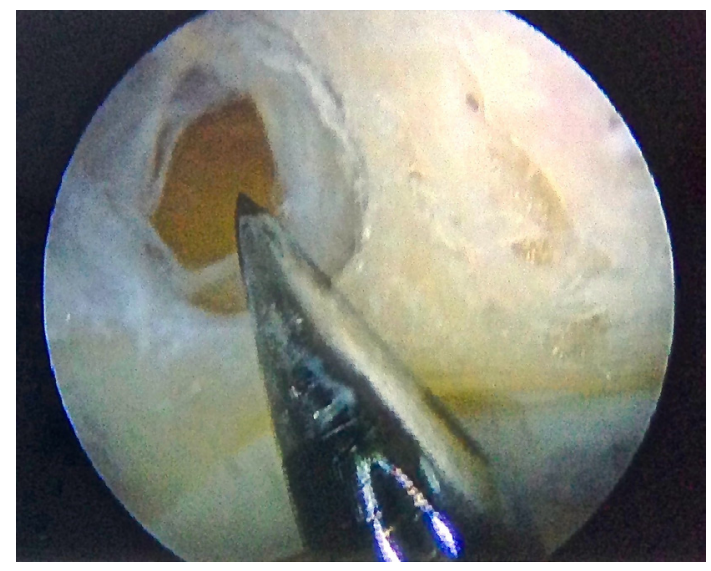

Figure 3: Cyst neck exposed after resection of ostrigonum.

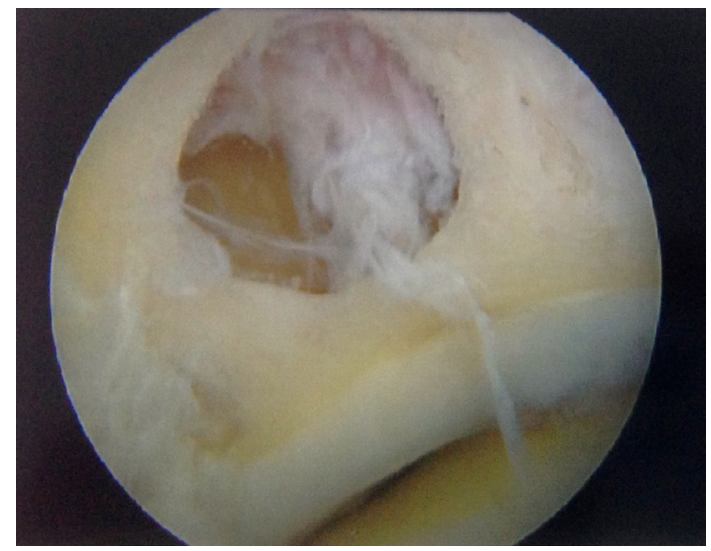

Figure 4: Fibrous tissue within the cyst.

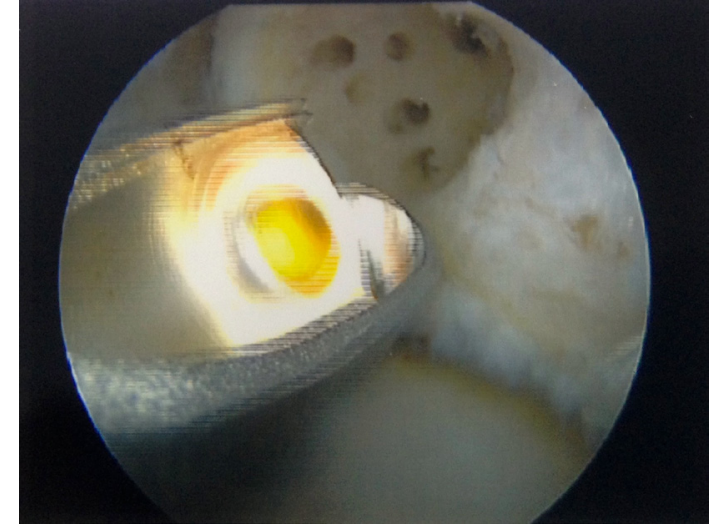

Figure 5: Base of the cysts following microfracture.

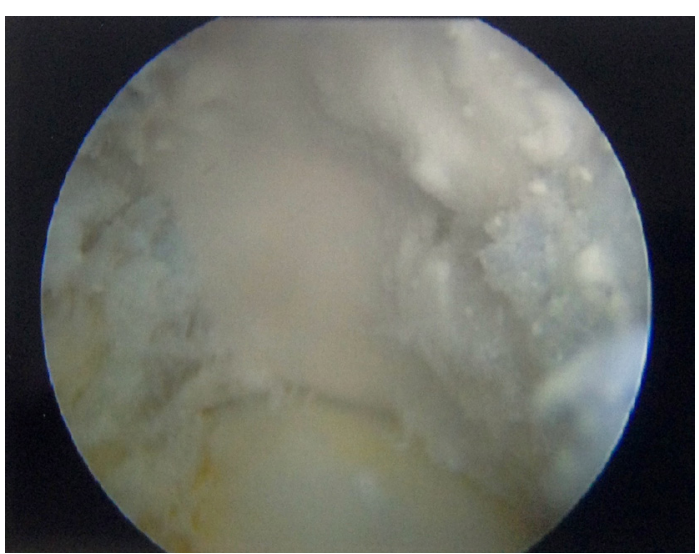

Figure 6: Cyst following grafting with an injectable bone graft substitute.

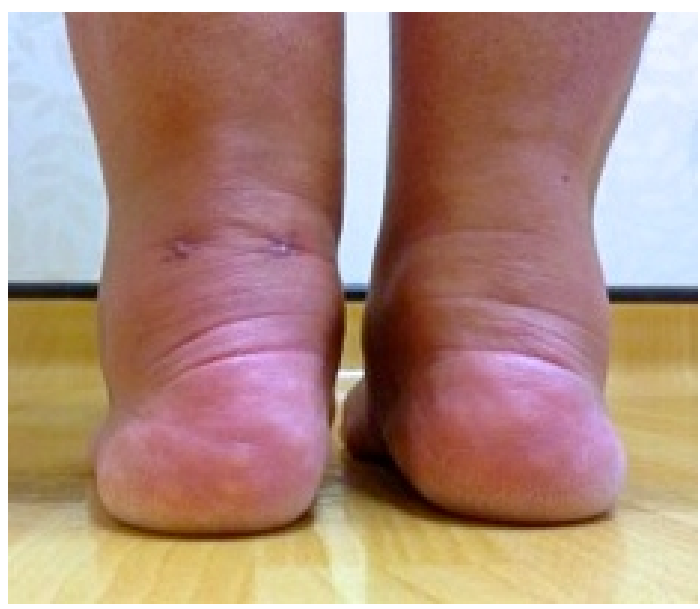

Figure 7: Hindfoot at 6 weeks post op (left side operated).

an injectable bone graft substitute (PRO-DENSE', Wright Medical) (Figure 6). The portals were closed and the patient was immobilized in a back slab, non-weight bearing for 2 weeks. She then went into a boot, fully weight bearing for a further 2 weeks. By 6 weeks the patient was already making excellent progress, with good portal healing and only a small amount of residual swelling (Figure 7). She was able to stand on tiptoes without pain (Figure 8) and was able to walk 45 minutes without 
Citation: Walker R, Nash WJ, Patel RS, Singh S (2014) Posterior Talar Intraosseous Ganglion Treated with Arthroscopic Debridement, Microfracture and Injectable Bone Graft Substitute. J Sports Med Doping Stud 4: 143. doi:10.4172/2161-0673.1000143

needing to rest. Even at this early stage she reported an improvement in her pain. Her 8 week post-operative X-ray showed some in-fill on the cyst (Figure 9) and the patient is doing very well and is able to walk a few hours a day without experiencing pain. She occasionally gets swelling but only for short periods of time.

\section{Discussion}

In 1972 Ogden and Griswold reported the first surgically managed case involving a unicameral bone cyst located in the anterior talar dome of a 15 year old [2]. This was successfully treated with curettage and autologous iliac crest bone grafting via an open antero-medial approach to the ankle. A year later Paaby reported two cases of talar intraosseous ganglions in adult patients, both successfully treated by open curettage and bone grafting with cancellous chips [3]. The first two cases of entirely arthroscopic treatment of a talar ganglion cyst were reported

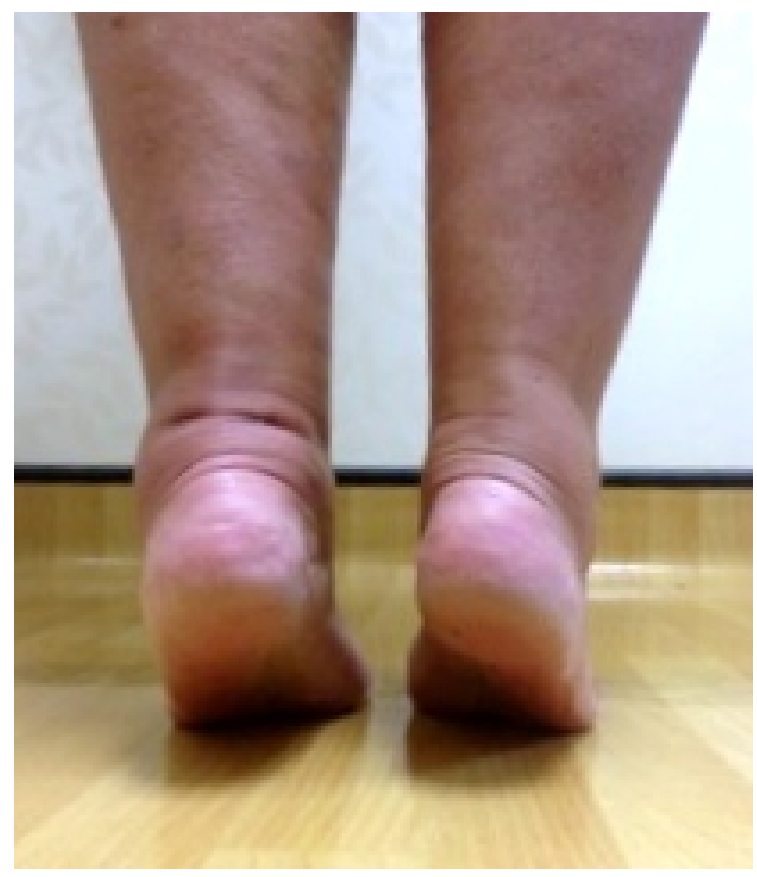

Figure 8: Full tiptoe possible at 6 weeks

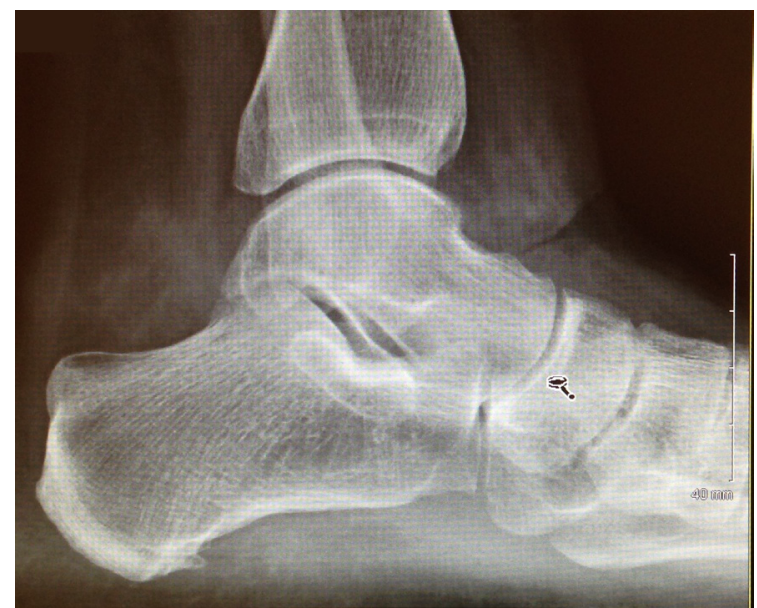

Figure 9: 8 week post-op X-ray showing some in-fill on the cyst. by van Dijk's group in 2003 and both cases utilized posterior ankle arthroscopy to debride and graft the ganglion cysts [8]. Subsequently two other authors have reported the technique. In 2011 Ogut et al. reported a small case series of 6 ankles in 5 patients, where posterior ankle arthroscopy was used to access and treat the posteriorly located talar cyst in a minimally invasive manner [7]. In these cases autologous bone was used to graft the defects in five ankles and hydroxyapatite in one. Good results were reported. In 2014 Dawe reported a single case study of posterior talar cyst treated with posterior ankle arthroscopy and injection of demineralized bone allograft in a gel, with good results at 3 years [6]. Due to the rarity of talarintraosseous ganglion cysts there are no studies in the literature comparing open versus arthroscopic methods of treatment or comparing the treatment methods of dealing with the cyst itself.

Another important feature in this case report was the utility of SPECT CT in the pre-operative assessment. There is increasing evidence supporting the use of SPECT CT for the diagnosis of non-specific or poorly localized musculoskeletal pain. This is particularly useful in the hands and feet where the number of small bones and proximity of joints can make the diagnosis of the true pain generator challenging [9]. In our practice we have found SPECT CT very useful and in this case study we believe that the SPECT scan correctly identified the talar cyst as the patient's main problem and allowed us to target treatment appropriately.

Compared with previously published cases, the technique presented here avoids the need for autologous bone graft to be taken, therefore reducing donor site morbidity by using an injectable bone graft substitute. This consisted of $75 \%$ calcium sulphate and $25 \%$ calcium phosphate. This bone graft substitute has been developed to treat benign bone cysts in areas where structural integrity is maintained by the surrounding bone. It acts as an osteo-conductive filler, curing in around 2 hours, and gradually resorbing over time. The calcium sulphate component resorbs more quickly, in theory leaving voids for vascular ingrowth. The other main advantage was the delivery method, as the bone graft substitute comes as a putty in a pre-filled syringe with an introducer, and will set hard even in a wet environment. These features make it very user friendly in the arthroscopic setting. Compared with demineralized bone matrix gel, PRO-DENSE ${ }^{\circ}$ cures hard in situ, which may facilitate earlier mobilization of the patient. There is little clinical data in publication regarding PRO-DENSE however one initial study of 24 patients being treated with the bone graft substitute after resection of benign bone tumours showed rapid biological integration with few complications [10].

In summary we feel that our technique builds on the principles pioneered by other authors. An arthroscopic approach to the cyst and the use of an injectable bone graft substitute which can cure in a wet environment is advantageous to both the surgeon and patient. Long-term follow up is now needed to establish whether the clinical improvement is sustained and that there is resorption of the bone graft substitute and subsequent bony infill of the cyst.

\section{Conclusion}

We present a case study which demonstrates the successful treatment of a posterior talarintraosseous ganglion using a posterior ankle arthroscopic approach and injectable bone graft substitute.

\section{References}

1. Hicks J (1956) Synovial Cysts in Bone. Aust N Z J Surg 26: 138-143.

2. Ogden J, Griswold D (1972) Solitary Cyst of the Talus a case report. J Bone Joint Surg Am 54: 1309-1310. 
Citation: Walker R, Nash WJ, Patel RS, Singh S (2014) Posterior Talar Intraosseous Ganglion Treated with Arthroscopic Debridement, Microfracture and Injectable Bone Graft Substitute. J Sports Med Doping Stud 4: 143. doi:10.4172/2161-0673.1000143

3. Paaby H (1973) Solitary Cysts of the Talus. Report of Two Operated Cases. Acta Orthop Scand 44: 560-563.

4. Barth E, Hagen R (1982) Juxta-Articular Bone Cyst. ActaOrthopScand 53: 215217.

5. Van Dijk CN (2004) Hindfoot Endoscopy for Posterior Ankle Pain. SpringerVerlag New York, Inc. Foot and Ankle Arthroscopy : 219-234.

6. Dawe EJ, Jukes C, Gougoulias N, Wee A (2014) Successful Arthroscopic Decompression and Synthetic Grafting of a Posterior Talar Cyst: A Case Report. J Foot Ankle Surg 20:35-36.
7. Ogut T, Seker A, Ustunkan F (2011) Endoscopic Treatment of Posteriorly Localized Talar Cysts. Knee Surg Sports TraumatolArthrosc 19: 1394-1398.

8. Scholten P, Altena M, Krips R, Niek van Dijk C (2003) Treatment of a Large IntraosseousTalar Ganglion by Means of Hindfoot Endoscopy. Arthroscopy 19: $96-100$

9. Huellner M, Strobel K (2014) Clinical Applications of SPECT/CT in Imaging the Extremities. Eur. J.Nucl. Med. Mol. Imaging 41: 50-58.

10. Evaniew N, Tan V, Parasu N, Jurriaans E, Finlay K et al. (2013) Use of a Calcium Sulfate-Calcium Phosphate Synthetic Bone Graft Composite in the Surgical Management of Primary Bone Tumors. Orthopedics 36: 216-222. 